\title{
Italian Army and Triple Alliance: An Historiographical Analysis
}

\section{Roberto Sciarrone}

Doi: 10.5901/mjss.2013.v4n4p197

Università La Sapienza di Roma

\section{Abstract}

During the twelve years between the taking of Rome by the conclusion of the Triple Alliance, the Italian army undergoes profound changes, as the policy of the newly unified state. These long twelve years to help us better understand the preparatory stages and the political effects that lead Italy to sign the agreements of the Triple Alliance. The politicians of taking an attitude almost detached, compared to the problems of the armed forces. In the financial crisis. The economic and social backwardness is manifested when we compare with other European countries. The problem that the Italian government has had to deal with the aftermath of Sedan, is transformation of the Italian army under the Prussian model.

Keywords: Triple Alliance, Italian Army, Prussian model, Battle of Custoza, German Empire, Kingdom of Italy, Deutscher Krieg, Austro - Prussian war, Third independence war, Sedan, Prussian military system

\section{Preamble}

Durante i dodici anni che separano la presa di Roma dalla stipulazione della Triplice alleanza, l'esercito italiano subisce profonde trasformazioni, come la politica del neo Stato unitario, ricca di colpi di scena e prodiga nel catapultarsi sul palcoscenico internazionale.

II morale dei militari italiani alla vigilia degli anni '70 dell'800 è sfiduciato, apatia e delusione serpeggiano tra i soldati. I fallimenti del 1866 mantengono vive le polemiche sugli insuccessi di Custoza e Lissa (Scotti, 2004), destinate a riemergere, nei dibattiti in Parlamento, ogni qualvolta si discute circa i progetti di riordinamento dell'esercito (Venosta, 1866).

Questi lunghi dodici anni ci aiutano a comprendere al meglio le fasi preparatorie e gli effetti politici che portano I'Italia a firmare gli accordi della Triplice alleanza.

I politici dell'epoca assumono un atteggiamento quasi distaccato, rispetto ai problemi delle forze armate.

In crisi le risorse finanziarie. L'arretratezza economica e sociale si manifesta non appena ci si confronta con gli altri Paesi europei.

Perciò il Parlamento si preoccupa, alla vigilia della guerra franco -prussiana (Shann, Delperier, 1991), di colmare il disavanzo economico e di limitare, di conseguenza, i bilanci della Guerra e della Marina.

L'impressione però che provocano gli avvenimenti in Francia sarà notevole in tutta Europa, scrive Fortunato Minniti: per trovare un riferimento con un fatto del nostro tempo che abbia rivestito la stessa importanza per $i$ contemporanei, si può paragonare Sedan a Hiroshima (Minniti, 1984). Gli ambienti militari europei reinterpretano le strategie di guerra. Le riforme, che nell'arco di sessant'anni aveva realizzato la Prussia, vengono messe in atto dagli altri Stati in poco tempo.

\section{Prussian military system and Italian army}

II problema che il governo di Giovanni Lanza ha dovuto affrontare, all'indomani di Sedan (Howard, 2001), è la trasformazione dell'esercito italiano secondo il modello prussiano (Blackbourn, 2003).

II nuovo ministro della Guerra Cesare Ricotti Magnani, in carica dal 7 settembre 1870 e presente su tutti i campi di battaglia del Risorgimento, non ha fama di riformatore ma possiede l'appoggio del generale La Marmora e del presidente del Consiglio Lanza.

II dibattito sulla leva di massa infuoca la scena politica italiana, corroborato dall'enorme effetto che suscitano le forze armate franco - prussiane per le dimensioni numeriche dei loro eserciti, ma una legge di reclutamento è sempre un fatto politico. Stabilire l'obbligo generale e personale del servizio militare significa far prestare servizio anche ai ceti borghesi, conseguenza necessaria che vede riconosciuta la propria importanza nelle leggi di ordinamento che sono 
presentate in Parlamento l'1 maggio 1867 e il 12 aprile 1869, la prima del generale Di Revel, la seconda del generale Bertolè - Viale.

II progetto Di Revel, è basato su analisi a cui giunge una commissione di studio appositamente creata. Le novità salienti del '67 sono l'abolizione della surrogazione ordinaria, la divisione dell'esercito in forze attive (325mila uomini più una riserva di 105mila) e presidiarie (140mila uomini) e l'istituzione di 30 Comandi di Distretto da poter mobilitare velocemente. La Camera però non può esaminare il progetto a causa delle vicende che porteranno a Mentana ed alla caduta del ministero Rattazzi.

In seguito, durante il 1869, il ministro Bertolé - Viale si occupa unicamente della questione reclutamento in base al quale l'esercito è portato a 425mila unità con una buona riserva di 200mila uomini, mantiene l'abolizione della surrogazione e introduce il volontariato di un anno.

Riassumendo, prima del 1870 è verosimile scoprire le fondamenta della successiva evoluzione dell'ordinamento militare, la guerra del '66 scatena l'opinione pubblica riguardo alle cause delle sconfitte subite e l'interesse che suscita l'annessione del Veneto. Scarso invece l'interesse per ciò che concerne temi squisitamente tecnici come la mobilitazione, i collegamenti e l'armamento.

L'approccio cambia dopo il 1870, l'opinione pubblica inizia a partecipare direttamente ai dibattiti circa le strategie e il ruolo dell'esercito, sono pubblicati numerosi opuscoli, saggi, articoli, pamphlet sulle varie regole di organizzazione difensiva, sulle possibili riforme di reclutamento, sulle strategie da attuare in base alle vicende internazionali, sono affrontati i grandi temi, dall'assetto difensivo nazionale ai problemi tecnici particolari.

Nei venti anni successivi, quindi, il dibattito politico e militare segue l'evoluzione delle riforme rispetto i tre medesimi temi: ordinamento, fortificazioni e strategia.

Nel 1870 l'Italia possiede un esercito privo di riserve addestrate, un sistema di mobilitazione che prevede il concentramento di tutte le truppe, sul teatro di guerra.

Insufficiente nell'affrontare crisi improvvise, e incapace quindi di mobilitarsi in tempi ristretti. Questi problemi diventano politici quando l'atteggiamento francese si fa più intimidatorio, è in gioco la sopravvivenza del neo Stato italiano e la classe dirigente e l'opinione pubblica pone al primo posto dell'agenda politica la riforma dell'ordinamento dell'esercito.

L'alternativa all'esercito a ferma breve o lunga è quella della Nazione armata che combatte l'esercito poiché istituzione stabile ma che sarebbe stata pronta a legittimarlo in camicia rossa o su base volontaria. II teorico della Nazione armata Luigi Amadei, ex comandante Corpo del Genio, introduce nel dibattito politico l'idea che le sole guerre giustificabili sono quelle per la difesa dell'indipendenza nazionale a differenza delle guerre combattute da eserciti permanenti a causa d'interessi politici divergenti tra gli Stati (Amadei, 1878).

In Volontari e Regolari di Paolo Fambri il modello della Nazione armata è criticato duramente, l'autore, infatti, sostiene che le teorie di Amadei possono essere applicabili solo là dove l'unità e l'indipendenza nazionale sono già consolidate, dove esistevano tradizioni militari stabili (Fambri, 1870).

Le considerazioni del Fambri furono quindi sposate pienamente dalla classe dirigente dell'epoca che scorge, nelle formazioni volontarie, un pericoloso canale di sbocco per rivendicazioni economiche, politiche e sociali che avrebbero potuto destabilizzare il Paese.

Il servizio militare obbligatorio si pone al centro del dibattito politico, il problema più importante da risolvere è quello delle pessime condizioni fisiche e morali degli uomini che si vogliono inquadrare. Mancano educazione morale, tattica e disciplina organizzata, la percentuale di analfabeti tocca il $60 \%$ negli anni tra il ' 66 ed il ' 75 , molti soldati non hanno ben chiari i concetti di unità nazionale e libertà, perciò secondo Carlo Corsi: a molti di loro bisognava insegnare a leggere $e$ scrivere, si deve iniziarli alla vita civile risvegliando in loro il senso della dignità umana (Corsi, 1881).

Grazie al rinnovato risveglio per le questioni militari che pervade l'opinione pubblica, durante il 1870 , il ministro Cesare Ricotti pressa in maniera sempre più insistente il Parlamento a deliberare sulle proposte da lui stesso portate all'attenzione delle camere. Inizia dal Senato, dove presenta la legge di ordinamento dell'esercito a modifica di quella del '54. Tre le novità sostanziali: il volontariato di un anno per gli studenti, la formazione di un esercito provinciale e l'introduzione dei limiti di età prefissati per ogni grado degli ufficiali in servizio attivo. II Senato approva i primi due punti e non il terzo Ricotti accetta suo malgrado che il provvedimento sia accettato attraverso un testo diverso rispetto l'originario anche per la fretta che la legge sia approvata in tempi rapidi da consentire l'introduzione, per la prima volta nella legislazione italiana, del principio riguardante il servizio personale obbligatorio, attraverso l'abolizione della surrogazione ordinaria e dell'affrancamento.

Dure critiche non mancano, soprattutto dagli ambienti della Destra ultra conservatrice capeggiata da Alfonso La Marmora che, nei suoi Quattro discorsi, manifesta il suo totale disappunto circa l'abolizione della surrogazione e 
dell'affrancamento per lui diritti insieme naturali ed acquisiti che bisogna rispettare per non spezzare le carriere liberali e danneggiare cosi l'economia di molte famiglie (La Marmora, 1871).

Ad ogni modo, di fronte all'urgenza di terminare la discussione del provvedimento prima della chiusura della sessione e del trasferimento dell'assemblea a Roma, destra e sinistra cercano di superare i contrasti riguardanti il servizio di leva obbligatorio, con promessa da parte del ministro di presentare l'anno successivo un nuovo progetto più completo.

La legge del 1854 prevedeva uno schieramento di prima linea pari a 250mila uomini, con la proposta di Ricotti si sarebbe dovuto alzare, tale soglia, sino a 300mila, aumentando quindi il contingente di prima categoria a 60mila uomini e riducendo la seconda attorno ai 30mila che sarebbe andata a formare parte dell'esercito di riserva. Seguendo il vecchio progetto Bertolé - Viale, Ricotti stabilisce inoltre una ferma di quattro anni per la fanteria e sei per la cavalleria su un totale di dodici e nove anni di disponibilità, la Camera propone tre anni per la fanteria ed una disponibilità di dodici anche per la cavalleria, si giunge ad un compromesso. La Camera, approva le richieste del ministro e lo autorizza a congedare anticipatamente le classi più anziane, con l'impegno di presentare l'anno successivo una nuova legge sul reclutamento che fissi la ferma a tre anni. Riassumendo è stabilito: l'introduzione del volontariato di un anno, arruolamento possibile dai diciassette anni, ammissione al volontariato per tutti coloro i quali avessero frequentato le scuole elementari fino alla quarta classe.

Le critiche più aspre si concentrano su due aspetti in particolare, le competenze troppo elementari che sono richieste per l'ammissione al volontariato e l'esclusione assoluta dell'intervento dello Stato per coloro che pur avendo i requisiti intellettuali, non possiedono mezzi finanziari per iniziare il volontariato. In Prussia, Austria e Francia, si prevedeva l'esenzione dalla tassa in caso di problemi economici della famiglia (Ritter, 1974), non in Italia, inoltre veniva contestato anche il basso livello d'istruzione.

Un altro argomento frequente, durante la discussione della legge sul riordinamento, è l'adozione di un veloce sistema di mobilitazione. Secondo Ricotti l'esercito andava organizzato come quello prussiano, dove bastava mandare un ordine o una circolare, perché tutti i corpi si organizzassero in modo rapido e all'unisono. L'esercito prussiano era organizzato attraverso un rigido ordinamento territoriale, in Italia era necessario istituire dei centri per smaltire l'enorme burocrazia, il ministro Ricotti, mediante Regio Decreto, istituisce quindi 45 Distretti che, secondo le previsioni dell'epoca, avrebbero dovuto ridurre i tempi ad otto giorni per l'esercito attivo e a quindici per quello provinciale.

La legge è approvata il 19 luglio 1870 dal Parlamento italiano riunito a Firenze in un clima di euforica soddisfazione, I'Italia festeggia così la prima legge militare moderna della sua storia. Ė inoltre formato il $20^{\circ}$ reggimento di cavalleria e rinforzato l'organico degli altri diciannove, non sono prese in considerazione, per il momento, possibili contromisure sul fronte fortificazioni, a tal proposito Domenico Farini "Aperta la frontiera orientale, aperta la frontiera verso la Svizzera, cioè senza ostacoli naturali in nostra mano, debolissimo, il tratto di frontiera verso la Francia [...] nulla sull'Appennino, nulla al di qua di questo, nulla o quasi se ne togliete Ancona e Gaeta, che ci metta in grado di prolungare la difesa (Minniti, 1984).

II Farini quindi riafferma un concetto strategico che più di dieci anni prima Luigi e Carlo Mezzacapo avevano proposto nei loro Studi topografici e strategici sull'talia, presupposta l'unità del Paese avevano indicato alcuni principi direttivi per la difesa della penisola, come lo sbarramento delle rotabili alpine, la loro difesa attiva, la scarsa possibilità degli sbarchi sulle coste del Sud (Ferrarelli, 1911).

La lunga serie di progetti incompiuti e di realizzazioni sommarie comincia con la presentazione al ministro della Guerra, il 2 agosto 1871, a dieci anni dall'inizio dei lavori, della Relazione a corredo del Piano generale di difesa dell'ttalia da parte della Commissione permanente per la Difesa generale dello Stato. Insediata fin dal 1862 e presieduta da Eugenio di Savoia Carignano, nel febbraio 1866 termina le indagini topografico - strategiche del territorio nazionale e redige un elenco dei luoghi che ritiene debbano essere fortificati, ma con l'annessione del Veneto è obbligata a riprendere i lavori per terminarli nel marzo 1868. La presa di Roma causa un analogo rinvio, fino al 2 agosto appunto.

Ricca di capillari descrizioni, la Commissione precisa le direttive principali attraverso le quali si sarebbe dovuto difendere il territorio italiano, divide quindi lo stesso in due scacchieri: continentale e peninsulare. Per la zona continentale, tutto il nord fino all'Appennino tosco - emiliano, è annunciata una difesa sistematica, per quella peninsulare una difesa circoscritta ad alcuni punti fortificati. La Relazione attribuisce grande importanza a Bologna come baluardo di difesa sistematica, mentre la difesa per capisaldi si sarebbe dovuta districare sulle piazze di Ancona e Lucera, sui campi trincerati di Roma e Capua e su alcuni porti fortificati come quello di Livorno, Civitavecchia, Gaeta e altri. Novantasette le piazze da guerra previste dal progetto, con una spesa di circa 146 milioni.

II piano ministeriale è studiato da una valida giunta parlamentare presieduta da Agostino Depretis, i lavori dureranno quasi due anni. La giunta affida al generale Bertolé - Viale il settore della difesa continentale e peninsulare, a Domenico Farini l'esame dei provvedimenti per l'armamento e la mobilitazione, la difesa alpina all'onorevole Teani e 
quella delle coste all'onorevole Maldini e allo stesso Depretis le ferrovie. Sullo sfondo, intanto, numerose pubblicazioni sull'argomento in questione, agitavano l'opinione pubblica e il dibattito politico si arricchiva di nuovi spunti di riflessione (Gandolfi, 1871).

La presa di Roma del 20 settembre 1870 e il trasferimento della capitale del Regno d'Italia da Firenze a Roma, 3 febbraio 1871, danno piena consapevolezza dei limiti strutturali dell'esercito ma infondono grande voglia di rinnovare l'apparato istituzionale e logistico dello stesso.

A gennaio del 1872 è presentata in parlamento dal Ministro Ricotti la legge sull'ordinamento dell'esercito e dei servizi dipendenti dal Ministero della Guerra. Approvata a settembre dell'anno successivo, la legge da una prima sistematica organizzazione alle riserve e crea un organo addetto allo studio e alla preparazione dei piani di guerra.

Sempre a settembre è approvata la legge sulla Circoscrizione militare territoriale del Regno. Sono predisposti i Comandi generali di Torino, Milano, Verona, Firenze, Roma, Napoli e Palermo e portati a sessantadue i vari Distretti sul territorio del Regno. Passano due anni prima che il Parlamento possa tornare a occuparsi di questioni attinenti la struttura organica dell'esercito. Intanto nel 1874 l'esercito conta 200.000 uomini suddivisi in dieci Corpi d'Armata, adatti più a una guerra di carattere difensivo per via della scarsa cavalleria e della non brillante artiglieria (Minniti, 1984)

Inoltre mancano i fucili per le riserve poiché i vecchi fucili trasformati nel 1867, circa 650.000 , sono in dotazione alle truppe di prima linea. Dei 300.000 Vetterly a retrocarica, che avrebbero dovuto essere pronti per il 1875 ne sono consegnati meno della metà poiché l'industria nazionale non è stata capace di soddisfare i tempi di consegna. Intanto alla Camera si apre la discussione su un terzo progetto di legge a modifica delle norme stabilite dalla 19 luglio 1871 , limitato a 16 articoli, siamo a marzo del 1875.

I punti più importanti dei disegni del '72 e del '73 sono ripresi ed è abolito il passaggio di categoria per i soldati semplici e per i volontari (Gallinari, 1978).

Dal 18 al 21 maggio 1875 inizia la discussione alla Camera sull'organizzazione della Milizia Territoriale, destinata a sostituire la Guardia Nazionale,organismo tattico, poiché se in Prussia ed in Austria il Landsturm, battaglione di riserva, esiste solo in casi di guerra estrema, in Italia in caso di conflitto questo terzo esercito, per via della inefficacia delle truppe di prima linea, è deputato a svolgere incarichi di presidio e mantenimento dell'ordine pubblico.

La legge, approvata, è salutata con grande euforia dal nuovo ministro della Guerra: il generale Luigi Mezzacapo e dal governo della Sinistra.

L'esercito ricevuto in eredità dal generale Mezzacapo è formato da dieci Corpi d'Armata su venti Divisioni più sedici reggimenti di Milizia per compiti presidiari. Tutti questi provvedimenti però, comparati a quelli delle altre nazioni europee, mostrano il carattere meramente difensivo del nostro esercito rispetto ai grandi numeri di Francia, Russia, Germania e Austria - Ungheria, superiori nei numeri e nella percentuale di spesa dedicata al bilancio di guerra.

Francesco Saverio Nitti afferma che le spese militari, ad ogni modo, favoriscono, seppur in maniera limitata, lo sviluppo economico dell'Italia settentrionale, dove da più di cinquanta anni vengono, in effetti, concentrate le più numerose unità dell'esercito e gran parte della Marina (Nitti, 1958).

A queste conclusioni giungono studiosi dell'epoca come Carlo Rovere (Rovere, 1877) e di quasi un secolo dopo come Federico Chabod che afferma: $E$ se la questione finanziaria costitui in ogni tempo e costituisce sempre un problema politico, esulando dal ristretto campo tecnico per investire tutta quanta la vita nazionale, in quel particolare momento di vita italiana essa diventava il problema politico; il problema nazionale per eccellenza, quello dalla cui risoluzione dipendeva l'essere stesso della nazione. Tutto si riconduceva e si riduceva lì: salvare il bilancio della nazione[... - e ancora, finisce lo storico - Vittorioso pertanto l'indirizzo Sella, la riorganizzazione dell'esercito ed il riarmo ebbero insufficiente appoggio finanziario. E fu, ripetiamo pure una necessità: ma ciò non toglie che dal punto di vista militare l'Italia rimanesse ancor più indietro delle altre grandi potenze, e che da tale situazione d'inferiorità troppo grande non ne venisse influenzata profondamente la sua politica estera, perché era difficile giocar serrato nel gioco diplomatico quando non s'aveva, alle spalle, la Home Fleet o la Guardia Prussiana (Chabod, 1965).

Alla luce di questa e di altre considerazioni si può affermare che il lavoro dei governi della Destra ha comunque colmato il disavanzo insieme con una costante e lungimirante politica di riarmo, considerazione che inserita nel quadro storico del neo - Stato italiano ha enorme peso riguardo alle difficoltà economiche che una nuova organizzazione politico - economica è destinata a superare durante il lungo processo post - unitario.

II compito della Sinistra, come rileva Ludovico Cisotti, sarà quello di preparare i cittadini alla partecipazione attiva, scrollando di dosso l'apatia politica che si respirava al tempo, preparazione intellettuale e morale degli italiani, compito arduo (Cisotti, 1865).

II dibattito politico, a sette anni dalla Triplice, inizia quindi a scaldarsi sulla questione difensiva - offensiva che l'esercito italiano, ricordiamo in piena fase di riordino istituzionale, deve mantenere. 
Molti i giudizi negativi circa le metodologie difensive, ma proprio questa, almeno nel 1875 , è la prospettiva che riscuote più successo tra i vari ministri del governo.

Ad ogni modo, con l'arrivo di Luigi Mezzacapo al Ministero della Guerra l'attività riformatrice delle istituzioni militari riprende intensa e costante.

L'ultima legge Ricotti sull'organizzazione della Milizia Territoriale è approvata, mentre nel gennaio del 1877 è proposto un progetto di riordinamento della Circoscrizione Militare Territoriale del Regno.

II progetto mira a creare tre nuove Divisioni Territoriali, oltre alle 17 già esistenti, per arrivare a due per Corpo d'Armata Territoriale; all'aumento del numero dei Distretti da sessantadue a ottantotto, dipendenti da venti Comandi superiori di Distretto. Durante la discussione del provvedimento Mezzacapo afferma il suo personale giudizio sull'operato del generale Cesare Ricotti che, a suo dire, va verificato e corretto dalle lacune esistenti, tra queste quella della scarsa preparazione ed efficienza delle truppe.

Attacchi più o meno forti non mancheranno di turbare il lavoro dei governi della Sinistra che tuttavia porterà avanti la realizzazione più importante: l'avvio dei lavori delle fortificazioni di Roma.

Infine si regola con una speciale Istruzione la mobilitazione della prima linea, alla prussiana per intenderci, nel marzo 1878 Mezzacapo lascia il Ministero.

II bilancio della sua attività è stato positivo per gran parte dell'opinione pubblica tedesca, la quale, riconoscendo all'esercito italiano una buona preparazione, tale da soddisfare i bisogni di una guerra, conseguita per di più in ristrettezza economica, ritiene una decisione saggia il sacrificare la costituzione di una riserva di mediocre efficienza al rafforzamento della prima linea.

II dibattito continua anche a causa delle dichiarazioni di Alois Von Haymerle, ex addetto militare austriaco a Roma, contenute nella pubblicazione Italicae res e riportate in prima pagina dalla stampa nazione (Ritter Von Haymerle, 1880).

II colonnello austriaco, infatti, nel descrivere la situazione politico - militare italiana, presupponendo intendimenti più o meno presunti tra governo e irredentisti, trae la conclusione che potrebbe essere imminente un attacco da parte dell'Italia per annettere i territori austriaci di lingua italiana. A confutare queste ipotesi è Luigi Mezzacapo, da poco comandante del Corpo d'Armata di Roma, 1879, che risponde al colonnello Haymerle attraverso le pagine della "Nuova Antologia" con due articoli. In Quid faciendum? (Mezzacapo, 1879) Mezzacapo traccia parte del programma militare della Sinistra di Crispi, definita dal Salvatorelli attivistica (Salvatorelli, 1939).

II neo comandante del Corpo d'Armata di Roma manifesta preoccupazione per la situazione internazionale e auspica la fine dell'isolamento politico italiano, criticando le numerose discussioni finanziarie del Paese che occupavano l'agenda politica del governo italiano. Insisteva sull'utilità di un esercito permanente e sull'inadeguatezza dei fondi destinati a tale scopo. Secondo Mezzacapo i provvedimenti da prendere sono: aumentare gli effettivi a seicento mila uomini, una dotazione sufficiente di armi, lo snellimento delle operazioni di mobilitazione, il mantenimento della ferma, un migliore trattamento economico degli ufficiali, la capitale totalmente fortificata e un maggior numero di stabilimenti marittimi e di roccaforti nella valle del Po.

Inoltre, scrive Mezzacapo, il nocciolo della questione sta nelle due sfortunate sconfitte di Custoza e Lissa, gravi disfatte che avrebbero traumatizzato e depresso tutti i discorsi successivi inerenti, le questioni sull'armamento nazionale (Mezzacapo, 1881). Rispetto alla gestione Ricotti sono questi i problemi posti in evidenza. Intanto è proseguita l'opera di riordino in campo amministrativo e organico e, sull'onda delle appena placate minacce di guerra con l'Austria, un Regio Decreto dell' 8 aprile 1880 regola la Milizia Territoriale in 300 battaglioni di fanteria e 100 compagnie da fortezza, mentre a giugno il Parlamento approva finanziamenti straordinari per ben ottanta milioni, distribuiti in cinque anni. Possiamo affermare che dal febbraio ' 77 al giugno '82 questo è l'unico intervento parlamentare in materia di riordino delle forze armate. Sicuramente un impegno finanziario di più piccola portata rispetto alla struttura pensata dal Mezzacapo, ma comunque un risultato soddisfacente per il neo Stato italiano. La stessa linea operativa è seguita di lì a poco dal nuovo ministro: il generale Ferrero. II suo primo atto è quello di chiamare alla presidenza del Comitato di Stato Maggiore il tenente generale Enrico Cosenz, che insieme al colonnello Luigi Pelloux darà vita a numerose innovazioni di carattere tecnico. II nuovo ufficio, in pochi anni, organizza un preciso sistema di preparazione organica e strategica e i primi piani di operazione. Inoltre sono costituiti altri due corpi d'Armata, che salgono così a 12 che conseguentemente provocano il parallelo aumento dei Comandi territoriali di Corpo d'Armata e di quelli superiori di distretto, con sede ad Alessandria, Torino, Milano, Piacenza, Verona, Bologna, Ancora, Firenze, Roma, Napoli, Bari e Palermo. L'aumento di potenza strategica perseguito dal Ministro Ferrero con tanta risolutezza costituisce dunque la dote, in termini di forza militare, che I'Italia porterà nella Triplice cui di lì a poco avrebbe aderito. Tornando alla politica il primo governo Depretis segna una svolta nella questione delle fortificazioni: nell'estate del 1877 il primo provvedimento proposto è di fortificare la capitale. Mezzacapo assegna il compito di sovrintendere ai lavori ed il comando della Divisione militare di Roma al tenente generale Giovan Battista Bruzzo, ingegnere militare. Da questo momento in poi la gran parte dei finanziamenti sarà 
destinata a Roma e alla sua fortificazione, nel dicembre 1878, infatti, è concessa dal Parlamento per le necessità dell'esercito la somma di dieci milioni di cui quattro per proseguire le fortificazioni già iniziate.

Intanto i problemi della difesa alpina monopolizzano l'interesse della pubblicistica militare. II saggio di Vittorio Emanuele Dabormida (Dabormida, 1878) critica la teoria della "manovra per linee interne" e sottolinea che l'uso del telegrafo ha annullato il fattore sorpresa e la presenza in campo di eserciti di massa fa sì che l'attaccante possa essere velocemente travolto. Secondo Dabormida il fattore decisivo è la velocità di spostamento e aggiunge che l'ipotesi più ragionevole è una ben organizzata resistenza sulle Alpi, con pochi uomini, di modo da concentrare la gran parte dell'esercito per lo scontro decisivo in pianura. Anche se, nelle conclusioni, Dabormida si abbandona a una riflessione non ottimistica scrivendo che in caso di guerra contro la Francia, l'unico risultato che l'Italia potrà raggiungere sarà quello di arrestare l'invasione. Un'altra interessante pubblicazione rispetto il tema trattato è Esame preliminare del teatro di guerra italo - austro-ungarico. Studio di Geografia Militare di Giuseppe Parrucchetti (Parrucchetti, 1878), nella quale l'autore esamina l'aspetto geografico militare di tutti i teatri di guerra che possono interessare l'Italia come la frontiera austriaca, svizzera e francese. L'Autore analizza diverse ipotesi di offensiva verso il Danubio e tende a escludere una guerra imminente contro l'Austria - Ungheria, mentre mostra una certa preoccupazione per l'altro versante, quello occidentale, dove diversi fattori tenderebbero a dare alcuni vantaggi logistici alla Francia. Le proposte esaminate tramite le pubblicazioni del periodo 1878 - 82 hanno numerosi punti di contatto, spicca soprattutto il condizionamento di una offensiva italiana sul fronte francese da parte di contemporanee operazioni tedesche sul Reno. Intanto l' 1 febbraio 1879 l'attività legislativa in materia di provvedimenti militari riceve nuovo impulso quando il ministro della guerra Mazé de la Roche e quello delle finanze Magliani, presentano alla Camera dei deputati sette disegni di legge, con previsioni di spesa di circa venticinque milioni per il conseguimento delle fortificazioni già iniziate, in particolare tre vanno a Roma, quattro alla difesa delle coste e ben diciotto ai valichi alpini dalle Alpi Marittime alla valle del Piave. Nell'ottobre dell' 80 il ministro Milon convoca il Comitato di stato maggiore generale e gli assegna lo studio di un nuovo piano di fortificazioni che subentri quello del ' 71 , dopo due anni verrà ultimata l'elaborazione e verranno riservati alle fortificazioni circa 55 milioni di lire. La questione finanziaria si colloca al primo posto nei dibattiti parlamentari a pochi mesi dalla firma dell'alleanza con l'Austria - Ungheria e l'Impero germanico, anche perché si era giunti a centoventotto milioni in cinque anni. In conclusione: nel 1871 si ha una forte azione iniziale che accelera l'evoluzione delle istituzioni militari, stabilendo serie premesse di un aumento delle forze mobili e di un esteso sistema difensivo permanente. Per quanto riguarda l'attività legislativa l'anno che segna una decisiva svolta è il 1875 , anno che vede riconfermato il ruolo principale dell'esercito attivo, poiché gli è affidato totalmente il dovere della difesa del Paese mentre l'arduo sistema delle fortificazioni, soprattutto per ragioni finanziarie, è divenuto già da due anni un mero stratagemma per consentire una sicura mobilitazione. E' sempre per giungere a questo scopo che si predispongono nuovi fondi fino al 1880, per un totale di centoquaranta milioni di lire dal 1872, per le opere di fortificazione e loro armamento. Poi, nel biennio 81'-82', la realizzazione di uno Stato Maggiore concretamente funzionante, l'adozione di un sistema difensivo stabile in rapporto ad una tattica offensiva e la decisa politica triplicista segnano una rilevante corrispondenza.

\section{Conclusions}

In questi dieci anni, dal 1871 al 1881, i dubbi e le incertezze che tormentano i pensieri dei politici italiani, circa le migliori alleanze da perseguire nel mutato ordine europeo, i continui dibatti che infuocano l'opinione pubblica riguardo la convenienza di un alleanza con la Germania o con la Francia non toccano in modo esclusivo le strategie dei vertici militari, affascinati dai successi prussiani nella campagna del 1866. L'aspetto più interessante e sconvolgente, che rilevano i vertici militari italiani, in quegli anni, è la rapidità tumultuosa con cui l'esercito francese, brillante in apparenza, crolla come un castello di carta all'irresistibile urto delle compagnie tedesche. Da queste immagini e dalla loro forza evocativa sarà interpretato e partirà un percorso d'imitazione del sistema militare prussiano, fortificato da una sempre più accesa vicinanza di politiche e intendimenti che porteranno all'alleanza con il nuovo impero. Alla luce di queste osservazioni vanno interpretati tutti gli atti e le decisioni della politica militare italiana all'indomani della guerra franco prussiana e dell'ingresso delle truppe nella città eterna.

II legame fra politica militare e politica estera è spesso ricordato in questi anni. In primis dal generale Ricotti che, un anno prima della svolta triplicista, chiede che sia reso noto, alla Camera, lo stato delle relazioni internazionali dell'Italia affinché i deputati possano orientarsi nel giudizio sui provvedimenti militari che sono stati sottoposti al loro esame. Questo legame tenderà a stringersi immediatamente dopo la stipulazione del trattato della Triplice alleanza, 20 maggio 1882 (Giordano, 2008). L'isolamento dell'Italia era stato spezzato. 


\section{Bibliography}

A. Gandolfi (1871), Bologna e l'Appennino nella difesa dell'Italia, Zanichelli

D. Blackbourn (2003), History of Germany, 1780-1918: The Long Nineteenth Century, Blackwell Publishing

F. Chabod (1965), Storia della politica estera italiana dal 1870 al 1896, Laterza

F. Minniti (1984), Esercito e politica da Porta Pia alla Triplice alleanza, Bonacci Editore

F. S. Nitti (1958), II Bilancio dello Stato dal 1862 al 1896-97, Laterza

F. Venosta (1866), Custoza e Lissa, fatti delle guerra italiana del 1866, Carlo Barbini Editore, Milano.

G. Ferrarelli (1911), Memorie militari del Mezzogiorno d'Italia, Laterza

G. Giordano (2008), Cilindri e feluche. La politica estera dell'Italia dopo l'Unità, Aracne

G. Parrucchetti (1878), Esame preliminare del teatro di guerra italo - austro-ungarico. Studio di Geografia militare

G. Ritter (1974), Frederick the Great: A Historical Profile, University of California Press

G. Scotti (2004), Lissa 1866. La grande battaglia per l'Adriatico, LINT Editoriale

L. Delperier (1991), French Army 1870-71 Franco-Prussian War (1): Imperial Troops, Osprey Publishing

L. Mezzacapo (1879), Quid faciendum?, ( In relazione all'opuscolo Italicae res di L. Von Haymerle, colonnello austriaco), in NA, $1^{\circ}$ ottobre 1879

L. Mezzacapo (1939), Armi e politica, in NA, 15 giugno 1881

L. Salvatorelli, La Triplice alleanza. Storia diplomatica 1877 - 1912, Istituto per gli Studi di politica internazionale, Milano, 1939

M. Howard (2001), The Franco-Prussian War, Routledge

V. E. Dabormida (1878), La difesa della nostra frontiera occidentale in relazione agli ordinamenti militari odierni, Edizioni Loescher

V. Gallinari (1978), Le riforme militari di Cesare Ricotti, in "Memorie storiche militari, Brossura editoriale 
